\title{
Analyzing the potential impact of BREXIT on the European research collaboration network $\odot$
}

Cite as: Chaos 30, 063145 (2020); doi: 10.1063/1.5139019

Submitted: 17 November 2019 . Accepted: 25 May 2020.

Published Online: 23 June 2020

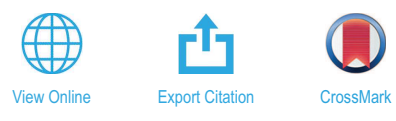

F. Bauzá, ' (D) G. Ruiz-Manzanares, ${ }^{2}$ L. Pérez-Sienes, ${ }^{3}$ (i) A. Tarancón, ${ }^{1,2}$ D. Íñiguez, 1,2,4 (iD) and J. Gómez-Gardeñes ${ }^{1,5,6, a)}$ (i)

\begin{abstract}
AFFILIATIONS
${ }^{1}$ Institute for Biocomputation and Physics of Complex Systems (BIFI), University of Zaragoza, 50018 Zaragoza, Spain

${ }^{2}$ Department of Theoretical Physics, University of Zaragoza, 50009 Zaragoza, Spain

${ }^{3}$ Grupo de Sistemas Complejos, E.T.S. Ingeniería Agronómica, Alimentaria y de Biosistemas, Universidad Politécnica de Madrid, 28040 Madrid, Spain

${ }^{4}$ ARAID Foundation, Diputación General de Aragón, 50018 Zaragoza, Spain

${ }^{5}$ Department of Condensed Matter Physics, University of Zaragoza, 50009 Zaragoza, Spain

${ }^{6}$ Center for Computational Social Science, University of Kobe, 657-8501 Kobe, Japan
\end{abstract}

Note: This article is part of the Focus Issue, Rare Events in Complex Systems: Understanding and Prediciton.

a) Author to whom correspondence should be addressed: gardenes@unizar.es

\begin{abstract}
In this work, we study the impact that the withdrawal of institutions from the United Kingdom caused by BREXIT has on the European research collaboration networks. To this aim, we consider BREXIT as a targeted attack to those graphs composed by the European institutions that have collaborated in research projects belonging to the three main H2020 programs (Excellent Science, Industrial Leadership, and Societal Challenges). The consequences of this attack are analyzed at the global, mesoscopic, and local scales and compared with the changes suffered by the same collaboration networks when a similar quantity of nodes is randomly removed from the network. Our results suggest that changes depend on the specific program, with Excellent Science being the most affected by BREXIT perturbation. However, the structure of the integrated collaboration network is not significantly affected by BREXIT compared to the variations observed after the random removal of institutions.
\end{abstract}

Published under license by AIP Publishing. https://doi.org/10.1063/1.5139019

The withdrawal of the United Kingdom (UK) from the European Union (EU), commonly known as BREXIT, will cause profound socioeconomical changes both in the UK and the rest of the EU. One of the foundational goals of the $\mathrm{EU}$ is to foster the collaboration of European actors at all levels. Perhaps, research is one of the fields that benefited the most from the creation of the EU, allowing alliances between research groups of different countries and disciplines. Here, we analyze the consequences of BREXIT in the network of collaboration among the EU institutions (research centers, industries, and universities) funded by the three different programs of the $\mathrm{H} 2020$ framework: Excellent Science, Industrial Leadership, and Societal Challenges. In particular, we study how different network metrics vary when UK institutions are removed and compare with null models in which a similar quantity of nodes is randomly removed from the network. In a nutshell, our results suggest that BREXIT effects vary across each of the three programs in which the H2020 framework is articulated. In particular, for the program Excellent Science, BREXIT produced a significant drop of both global and local network efficiency rooted on the central role played by UK institutions in this program. Nevertheless, for the aggregated network encoding all the collaborations across the three programs, we only find significant changes at the local level, in which the collaboration network after BREXIT appears more cohesive than expected. Finally, the mesoscopic properties of the new networks reveal that, while communities vary, the reorganization after BREXIT does not differ much from that expected randomly.

\section{INTRODUCTION}

One of the most useful frameworks to understand and characterize the functioning of many complex systems such as the Internet, power grids, or biological circuits is network theory. ${ }^{1-}$ 
Networks provide a useful and versatile representation of the backbone of interactions among the elements of complex systems in which statistical physics ${ }^{4,5}$ and dynamical system ${ }^{6,7}$ tools can be accommodated to study their structural and functional properties.

One of the most paradigmatic contributions of classical statistical physics tools to modern network science is the use of percolation theory ${ }^{8}$ to analyze the robustness of complex systems under perturbations. This application was spurred by the finding that scale-free networks are extraordinarily robust under the random deletion of nodes, i.e., failures, but very fragile to attacks, i.e., the targeted removal of highly connected vertices. A number of works addressed the analytical study of this important finding using percolation theory, ${ }^{10-12}$ allowing to connect the percolation threshold with the moments of the degree distribution of the underlying topology. In parallel, network robustness was also tackled from the dynamical systems' perspective by studying sandpile models mimicking the propagation of failures in the form of cascades across the network. ${ }^{13-16}$

Apart from its theoretical interest, the fragility of real world networks ${ }^{17-20}$ motivated an intensive search of efficient protection $^{21-24}$ and control ${ }^{25}$ methods for networked architectures. This interest has been boosted in the last few years motivated by the study of multilayer networks. ${ }^{26}$ This framework has revealed that real networked systems, when coupled (as, for instance, the Internet and the power grid), are much more fragile than when considered separately. ${ }^{27,28}$ This fragility is manifested by the change in the order of the percolation transition that pass from a smooth one for uncoupled systems (thus with early-warnings about the breakdown of the system), to an explosive one ${ }^{29}$ when coupled. ${ }^{30-34}$

In this work, we analyze the robustness of the European research collaboration network under BREXIT. Many papers and studies have been published about the impact that BREXIT would produce at different levels and sectors, such as, for example, on the EU Budget ${ }^{35}$ on agri-food policies, ${ }^{36}$ or on the pharmaceutical industry. ${ }^{37}$ The fostering of the collaboration of European actors being one of the foundational goals of the EU, research is one of the fields that benefited the most since the creation of the EU. The successive Framework Programs (FPs) have played a key role in this fostering and contributed decisively to the strengthening of scientific and technological collaboration in Europe, from the first FP established in 1984 with a budget of nearly $4 \times 10^{9} €$ to the present Horizon 2020 program with a budget of $80 \times 10^{9} € .^{38-40}$

Different publications have used network theory to analyze the collaboration ecosystem formed by the entities participating in these FPs. Protogerou et al. ${ }^{41}$ study the nature and structure of some of these policy-driven collaborative research networks, in particular those formed under the fourth, fifth, and sixth FPs in the area of Information Society Technologies. They find that these networks display characteristics of complex networks such as small-world property and scale-free distributions, and that they are structured around a core of organizations, mainly universities and research centers, which have assumed a very influential role over time. The EU Commission itself has also commissioned studies such as Refs. 42-44, where the authors implement measures similar to the ones used here. In Ref. 45, the authors found these scale-free networks to play an important role in generating and, especially, in diffusing knowledge by attracting key industry actors and by strengthening overall network connectivity through public support. In Ref. 46, the authors study how incentives for collaboration shape collaborative behavior and researcher productivity in the context of EU-funded research networks, observing that collaborations formed to capitalize on funding opportunities, while not effective in enhancing research productivity in the short run, may be an important promoter of effective collaborations in the longer run.

Here, we adopt the network perspective to studying BREXIT by considering this event as a targeted attack to the graph. To this end, we compare the effects of this attack with those of a random failure in which a similar number of entities are removed. The article is organized as follows. First, in Sec. II, we describe the dataset and the assumptions considered to construct the network of European projects (Sec. II A), and the different metrics considered to quantify the impact of BREXIT (Sec. II B). In Sec. III, we show the main results by analyzing the impact of BREXIT at global, mesoscopic, and local scales. Finally, in Sec. IV, we round off the article by discussing the results and giving some concluding remarks.

\section{NETWORK CONSTRUCTION AND ANALYSIS}

In this section, we introduce the way of defining the networks of European projects and the tools used to characterize the effect of the removal of nodes corresponding to UK institutions from the former graphs.

\section{A. Network formulation}

The data used to construct the collaboration networks of European projects have been obtained from the public EU H2020 database $^{47}$ spanning a time period from 2014 to February 2018. After processed by Kampal Data Solutions ${ }^{48}$ (a spin-off from the University of Zaragoza), we focus on the projects belonging to the three main programs of the $\mathrm{H} 2020$ framework: Excellent Science (P1), Industrial Leadership (P2), and Societal Challenges (P3). For each single project, we have obtained the following information: Project ID, the corresponding program, the institution acting as coordinator (name, Id, country, and the allocated funds), and the rest of institutions participating in the project (names, IDs, countries, and the corresponding allocated funds). In Table I, we summarize the main information for each of the three programs while in Fig. 1, we show the collaboration networks belonging to the three programs highlighting the nodes corresponding to UK institutions. We would also like to remark the different types of activity developed by the 19200 institutions studied in this work: 12265 (64\%) institutions correspond to private companies, 2010 (10\%) to research organizations, 1486 (8\%) correspond to public entities, 1481 (8\%) to higher education institutions, and 1962 (10\%) institutions correspond to other activity types.

With these data at hand, network construction is based on the collaboration among institutions through each of the projects. Thus, each node in the network corresponds to an EU institution and two nodes are connected by a link if the corresponding institutions have participated in the same project and, importantly, one of the two nodes being the coordinator of the shared project. This way, each project is represented as a star graph centered at the coordinator. 
TABLE I. For each program, we show the number of institutions that participate in at least one project, how many of these institutions are from UK, the total number of projects in each program, the number of projects with at least one participant from UK, the total amount of funds allocated in the programs, and the total amount of funds allocated to UK institutions.

\begin{tabular}{lccc}
\hline \hline & $\begin{array}{c}\text { P1 Excellent } \\
\text { Science }\end{array}$ & $\begin{array}{c}\text { P2 Industrial } \\
\text { Leadership }\end{array}$ & $\begin{array}{c}\text { P3 Societal } \\
\text { Challenges }\end{array}$ \\
\hline $\begin{array}{l}\text { Total number of } \\
\text { institutions }\end{array}$ & 3764 & 9498 & 12227 \\
$\begin{array}{l}\text { UK institutions } \\
\text { Proportion }\end{array}$ & 396 & 941 & 1184 \\
$\begin{array}{l}\text { Total number of } \\
\quad \text { projects }\end{array}$ & $11 \%$ & $10 \%$ & $10 \%$ \\
$\begin{array}{l}\text { Projects with UK } \\
\text { participation }\end{array}$ & 8249 & 4366 & 3870 \\
$\begin{array}{l}\text { Proportion } \\
\text { Total funds (Mill } € \text { ) }\end{array}$ & 2696 & 1083 & 1449 \\
$\begin{array}{l}\text { Funds UK institutions } \\
\quad \text { Mill } € \text { ) }\end{array}$ & 1934 & $25 \%$ & $37 \%$ \\
$\quad$ Proportion & $20 \%$ & 6257 & 9723 \\
\hline \hline
\end{tabular}

Therefore, the whole collaboration network is the sum of these start graphs and, as a consequence, it is a weighted graph.

We used two different approaches for the calculation of the weight $w_{i j}$ of a link connecting institutions $i$ and $j$. In the first one, the weight of a link is simply the number of projects shared by both nodes in which one of them has been the coordinator. The second approach consists in assigning the weight as the sum, over all projects involving both nodes (again when one of them has acted as coordinator), of the allocated funds to the node which has the role of participant. The results obtained for both approaches are qualitatively similar. We also obtained results for the network construction taking into account only the binary structure of the edges, i.e., the unweighted network. Results are also qualitatively similar, with the only difference that for the unweighted network, the changes after removing UK nodes differ from the set of networks after random removal of nodes in a less significant way than for weighted networks.

In order to avoid redundancy in the results and conclusions, the target of this work focused on the networks obtained with the first approach for weight calculation, taking into account only the number of projects without funds.

\section{B. Network descriptors}

Using the former network construction, we obtain four undirected and weighted networks, one for each program and a fourth one consisting in the aggregation of the other three. In Table II, we report the main properties for each network. To estimate the importance of each country to each of the four collaboration networks and identify the relative position that UK institutions have on these graphs we have computed, taking into account only the Giant Cluster Component (GCC), three usual centrality indicators: Betweenness (BC), Closeness (CC), and Eigenvector (EC) centralities, ${ }^{1-3}$ for each node in the GCC:
P1 Excellent Science

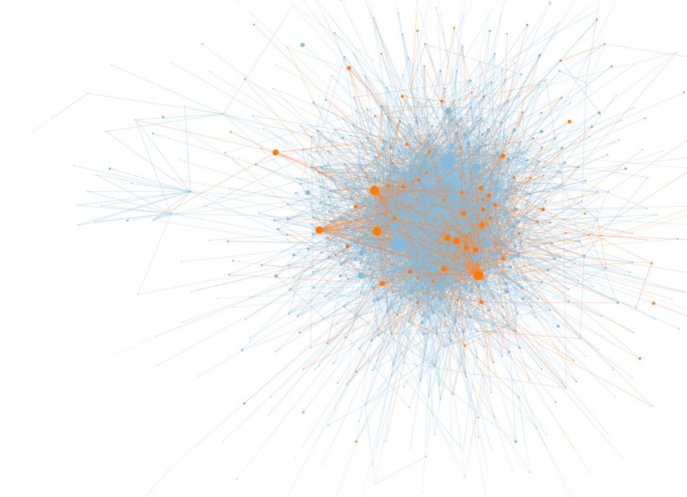

P2 Industrial Leadership

P3 Societal Challenges

UK Institutions

Non UK Institutions

FIG. 1. Collaboration networks of European projects belonging to the three main $\mathrm{H} 2020$ programs. For the sake of clarity, the networks only include the $10^{3}$ institutions with the largest number of collaborations. The nodes corresponding to UK institutions are highlighted in orange (see legend).

- Betweenness Centrality quantifies the importance of a node as product of its presence in the shortest paths connecting other pairs of vertices in the network. In particular, the $\mathrm{BC}$ of a node $v$ is computed as

$$
B C(v)=\sum_{s \neq t \neq v} \sum_{t \neq s \neq v} \frac{\sigma_{s t v}}{\sigma_{s t}},
$$


TABLE II. Main properties of the networks analyzed. For each network, we show the number of nodes (institutions), $N$, the size of the giant connected component, GC, the average degree, $\langle k\rangle$, the average strength per node, $\langle s\rangle$, and the diameter, $D$.

\begin{tabular}{lcccc}
\hline \hline Network & P1 & P2 & P3 & Aggregated \\
\hline$N$ & 3764 & 9498 & 12227 & 19204 \\
$G C$ & 3560 & 6670 & 10382 & 16512 \\
$\langle k\rangle$ & 4.70 & 2.58 & 3.41 & 4.16 \\
$\langle s\rangle$ & 5.24 & 2.84 & 3.70 & 4.79 \\
$D$ & 12 & 12 & 10 & 12 \\
\hline \hline
\end{tabular}

where $\sigma_{s t}$ is the number of shortest paths from node $s$ to node $t$ and $\sigma_{s t v}$ is the number of these paths that pass through $v$.

- Closeness Centrality points out the centrality of a node with respect to the distance from this node to the rest of nodes. The calculation of CC of node $v$ is as follows:

$$
C C(v)=\frac{1}{\frac{\sum_{s \neq v} d_{v s}}{N-1}}=\frac{N-1}{\sum_{s \neq v} d_{v s}},
$$

where $d_{v s}$ is the distance of the shortest path from node $v$ to node $s$ and $N$ is the total number of nodes. In other words, Closeness Centrality of node $v$ is the inverse of the average distance from $v$ to all other nodes.

- Eigenvector Centrality measures the importance of a node as proportional to the importance of its neighbors. This definition yields a recursive relation for the calculation of EC that leads to the calculation of the leading eigenvector, $\vec{x}$, of the Adjacency matrix A,

$$
\mathbf{A} \vec{x}=\lambda \vec{x}
$$

where $\lambda$ is the largest eigenvalue. Once solved, the $i$ th component of the resulting eigenvector $\vec{x}\left(x_{i}\right)$ accounts for the EC value of node $i$.

These three properties are usual estimators ${ }^{42,55,56}$ of the importance of institutions and countries in the spread of collaborations across research networks and the formation of new projects.

After calculating the three centrality values of all nodes belonging to the GCC of each network, we have estimated the importance of a country $\alpha$ according to a centrality measure $X, X^{\alpha}$, as the sum of the centralities $X_{i}$ of each node $i$ belonging to this country,

$$
X^{\alpha}=\sum_{i \in \alpha} X_{i} .
$$

Since we are interested in the relative position of UK institutions with respect to other countries $\alpha$, we have renormalized each value $X^{\alpha}$ by the centrality of the most central country according to the specific centrality measure $X: \tilde{X}^{\alpha}=X^{\alpha} / \max _{\beta}\left(X^{\beta}\right)$. In Table III, we report the ranking of each country according to the different values $\tilde{X}^{\alpha}$ for each of the four collaboration networks.

In addition, we have computed the average values of the centralities for the nodes belonging to UK and for the whole set of nodes. In Table IV, we can observe the ratio of these two averages for each centrality and for each of the three program networks and the aggregated one. This calculation gives us a more detailed information about the average importance of the individual UK nodes with respect to the other individual nodes. Taking the average value, we are eliminating the dependence of the centralities on the number of nodes of UK. That way, Table III is related to the global effect of BREXIT in the networks (taking into account the number of institutions in UK) and Table IV is related somehow to the relative effect

\begin{tabular}{|c|c|c|c|c|c|c|}
\hline \multirow[b]{2}{*}{ Rank } & \multicolumn{3}{|c|}{ P1 Excellent Science } & \multicolumn{3}{|c|}{ P2 Industrial Leadership } \\
\hline & $\mathrm{BC}$ & $\mathrm{CC}$ & $\mathrm{EC}$ & $\mathrm{BC}$ & $\mathrm{CC}$ & $\mathrm{EC}$ \\
\hline 1 & France (1.0) & Germany (1.0) & Germany (1.0) & Germany (1.0) & Germany (1.0) & Germany (1.0) \\
\hline 2 & UK $(0.67)$ & UK (0.84) & UK (0.99) & Spain $(0.57)$ & Spain $(0.78)$ & Spain $(0.65)$ \\
\hline 3 & Italy $(0.56)$ & France $(0.75)$ & France $(0.71)$ & France $(0.54)$ & Italy $(0.70)$ & France $(0.63)$ \\
\hline 4 & Germany $(0.41)$ & Italy $(0.71)$ & Italy $(0.64)$ & Italy $(0.41)$ & France $(0.68)$ & Italy $(0.58)$ \\
\hline 5 & Switzerland (0.34) & Spain $(0.70)$ & Spain $(0.51)$ & Belgium $(0.26)$ & UK (0.64) & UK (0.49) \\
\hline 6 & Netherlands (0.32) & Netherlands $(0.40)$ & Netherlands (0.48) & UK (0.23) & Netherlands (0.37) & Belgium (0.32) \\
\hline \multirow[t]{2}{*}{7} & Spain $(0.24)$ & Belgium (0.25) & Switzerland (0.32) & Greece $(0.23)$ & Belgium (0.33) & Netherlands (0.30) \\
\hline & \multicolumn{3}{|c|}{ P3 Societal Challenges } & \multicolumn{3}{|c|}{ Aggregated Network } \\
\hline Rank & $\mathrm{BC}$ & $\mathrm{CC}$ & EC & $\mathrm{BC}$ & $\mathrm{CC}$ & $\mathrm{EC}$ \\
\hline 1 & Germany (1.0) & Germany (1.0) & France (1.0) & Germany (1.0) & Germany (1.0) & Germany (1.0) \\
\hline 2 & France $(0.74)$ & Spain $(0.83)$ & Germany (0.93) & France $(0.95)$ & Spain $(0.77)$ & UK (0.80) \\
\hline 3 & Spain (0.69) & Italy $(0.83)$ & Italy $(0.76)$ & Spain $(0.53)$ & Italy $(0.76)$ & Italy $(0.72)$ \\
\hline 4 & UK (0.64) & UK $(0.81)$ & Spain $(0.73)$ & Italy $(0.48)$ & UK (0.74) & France $(0.71)$ \\
\hline 5 & Italy $(0.63)$ & France $(0.71)$ & UK (0.65) & UK $(0.41)$ & France $(0.71)$ & Spain (0.69) \\
\hline 6 & Netherlands (0.62) & Netherlands (0.55) & Belgium (0.58) & Netherlands (0.35) & Netherlands (0.48) & Netherlands (0.47) \\
\hline 7 & Greece $(0.41)$ & Belgium (0.46) & Netherlands (0.55) & Greece (0.25) & Belgium (0.38) & Belgium (0.34) \\
\hline
\end{tabular}

TABLE III. We report the ranking of the most central countries in the four collaboration networks according to three centrality measures: Betweenness (BC), Closeness (CC), and Eigenvector $(\mathrm{EC})$ centralities. We also show the values $\tilde{X}^{\alpha}$ for each country (see text for details). The position of UK institutions is highlighted in boldface. 
TABLE IV. Table showing the ratios with errors, between the average centrality values $(B C, C C$, and $E C)$ of the UK nodes and the average values of whole nodes, for the three program networks and the aggregated network. In the case of Closeness Centrality (CC), we have subtracted 1.0 to the average ratio to show a more fine comparison, due to the similarity of UK average value and whole network average value. Errors have been calculated by propagating the error of centralities average.

\begin{tabular}{lcccr}
\hline \hline Network & P1 & P2 & P3 & Aggregated \\
\hline$\frac{\langle B C\rangle_{U K}}{\langle B C\rangle_{\text {Network }}}$ & $1.4 \pm 0.4$ & $0.6 \pm 0.1$ & $1.1 \pm 0.2$ & $0.9 \pm 0.2$ \\
$\frac{\langle C C\rangle_{U K}}{\langle C C\rangle_{\text {Network }}}-1$ & $2 \times 10^{-2} \pm 1 \times 10^{-2}$ & $8 \times 10^{-3} \pm 7 \times 10^{-3}$ & $3 \times 10^{-3} \pm 5 \times 10^{-3}$ & $9 \times 10^{-3} \pm 5 \times 10^{-3}$ \\
$\frac{\langle E C\rangle_{U K}}{\langle E C\rangle_{\text {Network }}}$ & $1.4 \pm 0.2$ & $0.9 \pm 0.1$ & $0.9 \pm 0.1$ & $1.2 \pm 0.1$ \\
\hline \hline
\end{tabular}

of removing UK nodes compared to the effect of removing other network nodes.

From Tables III and IV, we observe that UK institutions clearly play a central role for the P1 program, they are on the top of the ranking, and their average centrality is greater than total average. However, for P2, P3, and the aggregated network, the position of UK institutions falls in the rankings and their average centrality values are not above network average, with the exception of the high ranking according to the EC in the aggregated graph, inherited from the high score reached by UK for this indicator in P1.

It is also important to remark the special case of the CC. Most of the nodes in each of the four networks have really similar values of average distance and, therefore, similar values of CC. This can be observed in Table IV where the average value for UK nodes and the total average value are very similar and implies that $\mathrm{CC}$ values for countries, shown in Table III, are really dependent on the number of institutions of each country in the GCC.

Finally, to characterize the structural changes suffered by the removal of UK research institutions, we focus on three main descriptors, namely, the global and local network efficiencies ${ }^{49,50}$ and the community structure. ${ }^{51}$ The first two metrics were originally designed to characterize the quality of information exchange among nodes of a network both at global and local scales, while the third one focuses on the mesoscale by analyzing the partition of the network into groups of nodes that are tightly connected.

\section{Global efficiency}

Global efficiency, $E^{G}(\mathcal{G})$, accounts for the ability of a network to connect any given pair of nodes. In particular, given a weighted graph $\mathcal{G}$ as those described above for European projects, the global efficiency is calculated as follows. First, weighted shortest paths are computed for each pair $(i, j)$ of nodes in $\mathcal{G}$. To this aim, we assume that the length $l_{i j}$ of an existing link $(i, j)$ is equal to the inverse of its weight $w_{i j}$. This way, we consider that the more projects two institutions are commonly involved in, the closer is their mutual relationship. With this proviso, the distance $d_{i j}$ between institutions $i$ and $j$ is defined as the sum of the link lengths over the shortest path connecting $i$ and $j$. Note that shortest in the context of weighted graphs refers to the path connecting $i$ and $j$ for which the sum of the link lengths is the minimum possible, i.e., regardless of the number of links crossed in this path. Finally, the efficiency $E_{i j}$ in the communication from $i$ to $j$ is assumed to be inversely proportional to the length of the shortest path, i.e., $E_{i j}=1 / d_{i j}$ and, correspondingly, the global efficiency of graph $\mathrm{G}$ is defined as the average of $E_{i j}$ over all the pairs of nodes,

$$
E^{G}(\mathcal{G})=\frac{1}{N(N-1)} \sum_{i, j \neq i} \frac{1}{d_{i j}} .
$$

Note that, according to this definition, in case there is no path connecting $i$ to $j$, we obtain $d_{i j}=\infty$, leading to a 0 value for the efficiency in the communication between these nodes.

\section{Local efficiency}

Local efficiency, $E^{L}(\mathcal{G})$, aims at capturing the robustness of a network under small scale failures. In particular, it measures the cohesiveness of the neighborhoods around single nodes in order to quantify how well information would be exchanged by the acquaintances of a node in the event the latter is removed from the network. To compute $E^{L}(\mathcal{G})$, one takes the average of all the global efficiencies of the $N$ subgraphs $\mathcal{G}_{i}$ resulting from considering the neighbors of each node $i$ and all their interconnections (excluding those with node $i$ ). As a result, the local efficiency of a network $\mathcal{G}$ is

$$
E^{L}(\mathcal{G})=\frac{1}{N} \sum_{i} E^{G}\left(\mathcal{G}_{i}\right) .
$$

Note that, for a complete (unweighted) subgraph $\mathcal{G}_{i}$ (where the neighbors of $i$ form also a complete graph), the Efficiency is maximum $E^{G}\left(\mathcal{G}_{i}\right)=1$. On the other hand, the minimum case appears when $\mathcal{G}_{i}$ is a star graph centered in $i$, since the absence of connections among the neighbors of $i$ leads to $E^{G}\left(\mathcal{G}_{i}\right)=0$.

\section{Community analysis}

Finally, in addition to the characterization of the networks at the global and local scales, we tackle their mesoscopic description by computing their partition into communities. ${ }^{51}$ Mesoscopic properties of a network refer to the features and effects that emerge from the interplay of nodes in groups whose size is certainly smaller than the whole network size.

In this work, the community structure is seen as a proxy of the fragmentation of the network, under the hypothesis that inside a community, it is easier to transmit information, spread knowledge, or get new partners for a future project. In case BREXIT produces a fragmentation originating a considerably bigger number of communities, it could be a symptom of the network fragility at this level. A similar approach can be seen in Ref. 44 where the authors use the concept of component, based on the assumption that a larger number of components is associated with higher fragmentation. 
The procedure used here to obtain the community structure is the partitioning method known as Louvain, ${ }^{52}$ which requires the size of the network and its weight nature. As many other methods in the network literature, ${ }^{51}$ that of Louvain is based on the optimization of modularity. The modularity of a network partition ${ }^{53}$ accounts for the fraction of edges that fall within the proposed groups minus the expected fraction if edges were distributed at random. For a weighted network $\mathcal{G}$, the modularity of a given partition into $n$ communities, $\left\{c_{1}, \ldots, c_{n}\right\}$, is given by

$$
Q\left(\mathcal{G} ;\left\{c_{1}, \ldots, c_{n}\right\}\right)=\frac{1}{2 m} \sum_{i, j}\left[w_{i j}-\frac{s_{i} s_{j}}{2 m}\right] \delta\left(c_{i}, c_{j}\right),
$$

where $s_{i}$ is the strength of node $i, s_{i}=\sum_{j} w_{i j}$, and $m$ is the total weight of the network $2 m=\sum_{i} s_{i}$.

Given a network $\mathcal{G}$, finding the exact partition with the maximum possible value of $Q$ by testing all possible partitions is not computationally feasible and hence heuristic optimization algorithms are needed. The Louvain method tackles the problem by focusing first on small communities in which modularity is maximized locally. These communities are subsequently transformed into single nodes that, in turn, form a new network (smaller than the original one) in which a similar local modularity optimization is implemented. The sequence of local optimizations produces nearly optimal partitions in a computationally fast way.

\section{Network comparisons}

As already introduced, our goal in this work is to quantify BREXIT effects in the network of European projects by comparing the before- and after-BREXIT networks through the computation of the former network descriptors. However, in order to assess whether the impact of BREXIT is significant regarding a network descriptor or not, it is not enough to compare the measurements made in the original and the perturbed networks. Then, we will compare the network without the UK nodes with a series of networks obtained by removing a similar quantity of European institutions chosen at random. The random node removal process is as simple as selecting $n$ nodes randomly from the network and removing these nodes, where $n$ is the number of nodes that belong to UK for each network. This way, the resulting (reduced) random network has the same number of nodes than the network without the UK nodes.

For this statistical comparison, we have generated a set of 500 random networks for each of the research collaboration network and computed the average values and the statistical median of the main structural descriptors after this set of random deletion of nodes.

Regarding the statistical significance, its computation is not straightforward in this case. We rejected the possibility of obtaining the significance by means of the statistical mean and standard deviation because of the non-Gaussian and non-symmetric form of the probability distribution (histograms) obtained from the random set of networks, as we can see in Sec. III. Therefore, we have assumed as statistical significance of a testing value (BREXIT value), given a probability distribution (obtained from evaluating the ensemble of random networks), one minus the probability of obtaining a value from the probability distribution, as extreme or more extreme than the testing value.
The significance defined above is computed as follows, given an ordered data sample and the testing value:

$$
s f c\left(x^{\prime}\right)=2 \times\left|\frac{n_{x^{\prime}}}{N}-0.5\right|,
$$

where $x^{\prime}$ is the testing value, $n_{x^{\prime}}$ is the number of data between $x^{\prime}$ and the lowest end of the data sample, and $N$ is the size of the sample. In order to be able to apply this formula, we assume that the numerical probability distribution (histograms obtained from the random sets of networks) is a proper representation of the real probability function. Therefore, this computation is equivalent to calculate the probability distribution area further from the median than the testing value, multiplied by two since we are interested in a two-sided test.

This significance goes from 0 when $x^{\prime}$ is equal to the median to 1 when $x^{\prime}$ is greater/less than the maximum/minimum of sample data. In our case, the testing value is the descriptor value for the network without UK nodes and the sample data are the descriptor values for the random set of networks.

While the former procedure allows evaluating the significance of many quantitative measurements (such as the change in the local and global efficiencies), the comparison between different network partitions demands more elaborated metrics. To this aim, here we make use of the Wallace index, ${ }^{54} \mathrm{~W}$, for measuring the similarity of two different partitions of the same set of $N$ objects (nodes in our case) in groups, communities, or clusters. Wallace similarity index is obtained by computing the pairs of nodes which belong to the same group (community) in each partition. In a general case, given two partitions, there are $T$ pairs of nodes that are together in the same group in both partitions, $P$ pairs of nodes that are together in the same group in the first partition, and $Q$ pairs of nodes that are together in the same group in the second partition. Given these numbers, the Wallace Index is expressed as follows:

$$
W=\sqrt{\frac{T}{P}} \cdot \sqrt{\frac{T}{Q}}=\frac{T}{\sqrt{Q \cdot P}},
$$

i.e., the geometric mean of the fraction $\frac{T}{P}$ of nodes that are in the same group in first partition are also in the same group in the second partition, and the fraction $\frac{T}{Q}$ of nodes that are in the same group in the second partition are also in the same group in the first partition.

In our case, we use the Wallace Index to measure the similarity between the partition into communities of the original network and that of the network without the UK institutions. We compare the similarity value $W$ obtained for each program and the aggregated network with the corresponding similarities median obtained comparing the original networks with those in which nodes are removed at random. Let us remark that we cannot measure the similarity of partitions for different sets of nodes; therefore, we only use the common nodes of the original network and the reduced ones (after the removal of UK nodes or a random subset of vertices) for the calculation of $W$.

\section{RESULTS}

Let us start by illustrating BREXIT effects at the global scale. From Fig. 2, we observe that the removal of nodes from a network 


\section{Original Without UK Median}

P1 Excellent Science

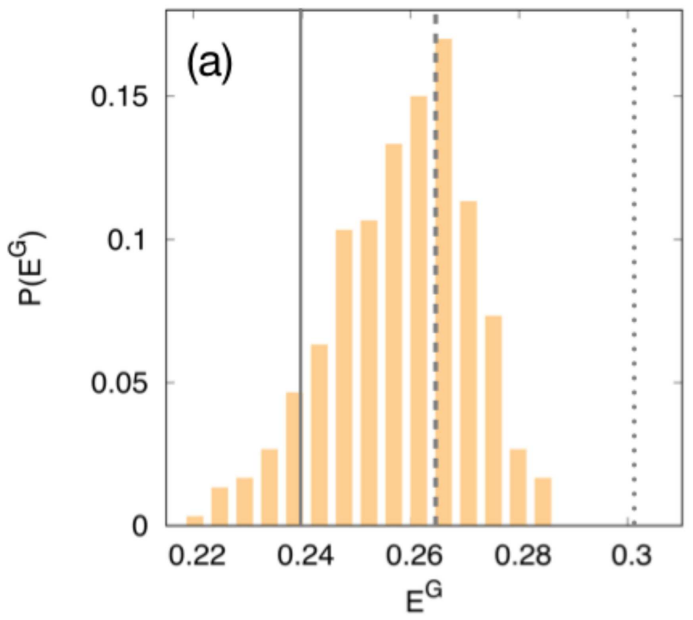

P3 Societal Challenges

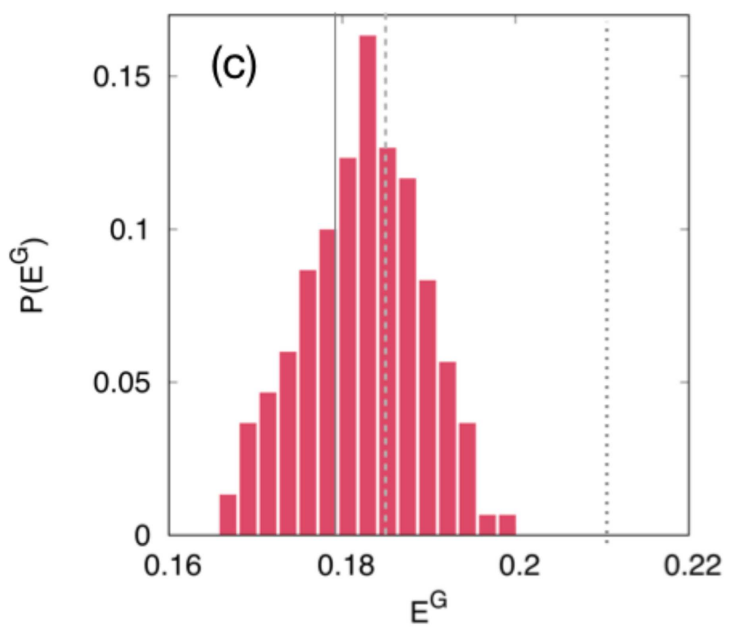

P2 Industrial Leadership

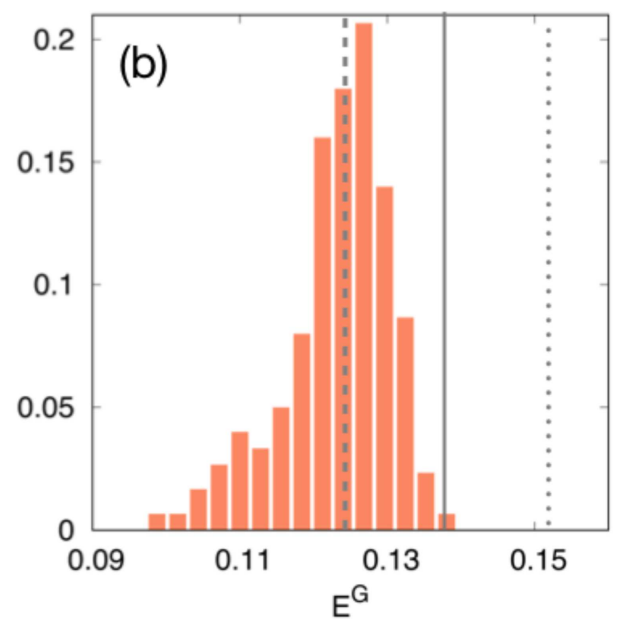

Aggregated network

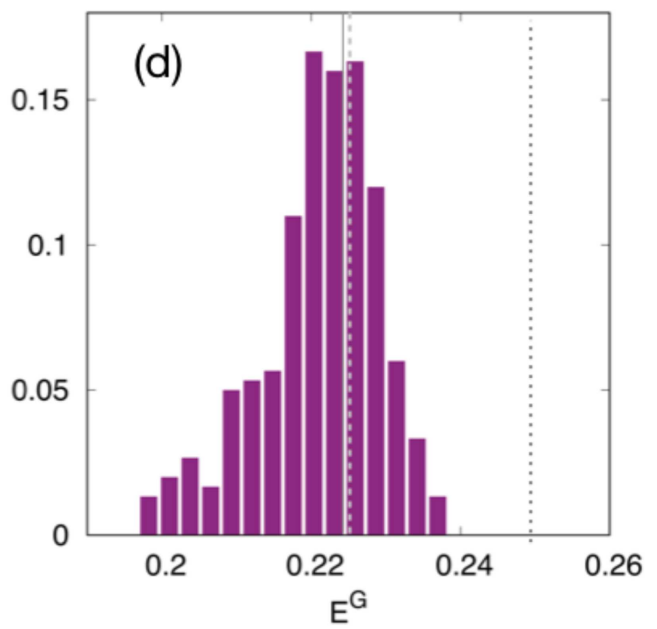

FIG. 2. Values of the global efficiency, $E^{G}$, for the original networks (dotted line), the collaboration graphs without UK institutions (solid line), and the median of the values for $E^{G}$ obtained when randomly removing a similar number of nodes to those removed after BREXIT in the corresponding networks. We also show the histogram of these latter values for a set of 500 networks for which a random deletion of nodes has been implemented. The plots correspond to program P1 (a), P2 (b), P3 (c), and the aggregated network (d).

leads to the decrease of global efficiency in the damaged networks with respect to the original one (dotted line). This decrease is due to the remotion of links attached to the deleted vertices, thus decreasing the global connectivity of the graph. However, the differences between the effects of BREXIT and random deletion of institutions depend strongly on the specific research program.

In particular, for P1 [Fig. 2(a)], the decrease in the global efficiency of BREXIT (solid line) is significantly larger $\left[s f c\left(E_{B R E X I T}^{G}\right)\right.$ 
$=0.85]$ than that produced randomly (dashed line). However, for P2 [Fig. 2(b)], the effect is the opposite, and the decrease of $E^{G}$ with respect to the original graph is significantly smaller $\left[s f c\left(E_{B R E X I T}^{G}\right)\right.$ $=0.95]$ for BREXIT network than that produced by random deletion of nodes. The different behavior for the change in $E^{G}$ in P1 and P2 is clearly connected to the different centrality of UK institutions in these networks. While for P1, UK nodes are on the top of the rankings, display high values for $\tilde{X}^{U K}$, and their average centrality values are greater than average network nodes, this is not the case for P2, pointing out that the deletion of UK institutions in P1 will cause the loss of more shortcuts than randomly expected while the opposite holds for P2.

Following the former reasoning and by looking the centrality rankings reached by UK institutions and their average centrality ratio in the P3 and aggregated networks, we can conclude that the deletion of UK institutions would not produce remarkable differences with respect to the deletion of randomly chosen nodes. The average $\mathrm{BC}$ of $\mathrm{UK}$ nodes in $\mathrm{P} 3$ is less than the network average value, but it is compensated by the $\mathrm{EC}$ and the opposite occurs for aggregated network, where the lower average EC is compensated by BC. This is confirmed in Figs. 2(c)-2(d) from which it is clear that BREXIT produces slightly less efficient networks than randomly expected but, clearly, the differences are not significant enough $\left[s f c\left(E_{B R E X I T}^{G}\right)=0.48\right.$ for P3], especially for the aggregated graph $\left[s f c\left(E_{B R E X I T}^{G}\right)=0.006\right.$ for aggregated graph] in which the two values for $E^{G}$ in the perturbed networks (BREXIT and random) are roughly the same.

The effects of nodes remotion in the local efficiency $E^{L}$ of a network is not as obvious as in the case of global efficiency, for which a decrease in $E^{G}$ is always expected. The outcome, in terms of local efficiency, depends strongly on the nature of the nodes removed. In particular, when the nodes removed and their original neighborhoods form dense subgraphs, their remotion leads to a strong decrease in $E^{L}$. On the contrary, when the nodes removed are mostly centers of star-like subgraphs, their deletion increases $E^{L}$ with respect to the original network.

In Fig. 3, we have plotted the effects produced by the targeted (BREXIT) and random deletion of nodes in $E^{L}$ for the tree specific programs and the aggregated collaboration network. Remarkably, in the four networks, the random remotion of institutions always yields (on average) a decrease in $E^{L}$. This change is significant for P1 [Fig. 3(a)], P2 [Fig. 3(b)], and P3 [Fig. 3(c)] networks $\left[s f c\left(E_{\text {OriginalNetwork }}^{L}\right)=0.75\right.$ for $\mathrm{P} 1, s f c\left(E_{\text {OriginalNetwork }}^{L}\right)=0.80$ for $\mathrm{P} 2$ and $s f c\left(E_{\text {OriginalNetwork }}^{L}\right)=0.71$ for P3], but almost negligible for the aggregated network [Fig. 3(d)] $\left[s f c\left(E_{\text {OriginalNetwork }}^{L}\right)=0.06\right]$. However, this is not the case for networks without UK institutions since the relative increment/decrease of $E^{L}$ depends on the specific program. In particular, for P1 (similarly to what was found for $E^{G}$ ), the network after BREXIT becomes significantly less locally efficient than randomly expected $\left[s f c\left(E_{B R E X I T}^{L}\right)=0.77\right]$. However, while for $P 2$, no significant $\left[s f_{c}\left(E_{B R E X I T}^{L}\right)=0.53\right]$ differences are found between BREXIT and the random remotion of nodes, for P3, the situation is the opposite to that of P1, pointing out that those subgraphs centered around UK institutions in P3 are much more sparse than the average $\left[s f c\left(E_{B R E X I T}^{L}\right)=0.91\right.$ for P3]. Finally, in the aggregated network $\left[s f c\left(E_{B R E X I T}^{L}\right)=0.97\right.$ for aggregated network] we find a similar scenario to that of $\mathrm{P} 3$ since, $\mathrm{P} 3$ being the largest specific network, its behavior according to $E^{L}$ dominates when integrating the three networks into the aggregated one.

Now, we focus on the reorganization of the collaboration network at the mesocopic scale. In Fig. 4, we show the size of the seven largest communities $\left(C^{\alpha}\right)$ in the four networks after BREXIT. The bar plots also show the composition of these largest communities in terms of the ten largest communities $\left(C^{\alpha \prime}\right)$ in the original networks. The new and original communities are also termed after the node (institutions) with the largest number of collaborations in the corresponding community. In addition, in Fig. 5, we report the values of the Wallace Index that compare the original partitions of the four networks with the new ones after BREXIT ( $\left.W^{B R E X I T}\right)$ and with those obtained in the set of networks where an identical number of institutions are removed $\left(W^{\text {random }}\right)$. The values from the set of random networks are represented by the histogram (the dashed line corresponds to the median) and the $W^{B R E X I T}$ is represented by the solid line.

In the first network $\mathrm{P} 1$, the deletion of the UK institutions makes the original largest community $C^{1 /}$ disappear, the largest fraction of its nodes being placed at the new community $C^{7}$. Besides, most of the nodes of the former community $C^{2 \prime}$ compose the new community $C^{3}$, while the third original community $C^{3 \prime}$ is roughly split between new clusters $C^{2}$ and $C^{5}$. Thus, from this picture, we conclude that the mesoscopic consequences of BREXIT in P1 is to break many original clusters to form the new ones.

For P2 and P3, the main original groups are retained more in the new partition after BREXIT, especially in the case of P3 for which the first and second largest communities $C^{1 \prime}$ and $C^{2 \prime}$ remain almost the same. The larger similarities for the new partitions in $\mathrm{P} 2$ and $\mathrm{P} 3$ are revealed also by comparing their respective values of $W^{\text {BREXIT }}$ with that for P1 $\left(W^{\text {BREXIT }}=0.35\right.$ for P $1, W^{\text {BREXIT }}=0.47$ for $\mathrm{P} 2$, and $W^{B R E X I T}=0.56$ for P3). However, the three values for $W^{\text {BREXIT }}$ do not display significative differences when compared to the corresponding values of $W^{\text {random }}\left[s f c\left(W^{B R E X I T}\right)=0.31\right.$ for P1, $s f c\left(W^{B R E X I T}\right)=0.28$ for $\mathrm{P} 2$ and $s f c\left(W^{B R E X I T}\right)=0.36$ for P3], pointing out that the removal of UK institutions, although changing the original partition, do not yield a different mesoscopic reorganization from those obtained by randomly removing nodes.

In the case of the aggregated network, although we cannot state that the $W^{B R E X I T}=0.44$ is significantly different from the random values $\left[s f c\left(W^{B R E X I T}\right)=0.52\right]$, we can observe that its significance is considerably larger than that of the individual programs. From Fig. 4, we observe that the largest community $C^{1}$ does not correspond to a single original community but is composed of a small fraction of the largest original community $C^{1 \prime}$ and part of the third most populated original cluster $C^{3 \prime}$. For the remaining new clusters, we observed that almost every new community can be identified with an original one: $C^{2}$ with $C^{1 \prime}, C^{3}$ with $C^{4 \prime}, C^{4}$ with $C^{5 \prime}, C^{5}$ with $C^{9 \prime}, C^{6}$ with $C^{2 \prime}$, and $C^{7}$ with $C^{7 \prime}$. However, especially in the case of $C^{2}, C^{3}$, and $C^{6}$, the former correspondences come together with a decrease in the size of the institutions from the original clusters.

In order to strengthen this similarity analysis, we also present a comparison (see Table V) for the number of communities and their size distribution in the partitions found for the network without UK nodes and the set of random networks, for each program. 


\section{Original}

Without UK

Median

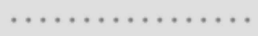

$\longrightarrow$ - - - - - - - - - - - -

P1 Excellent Science

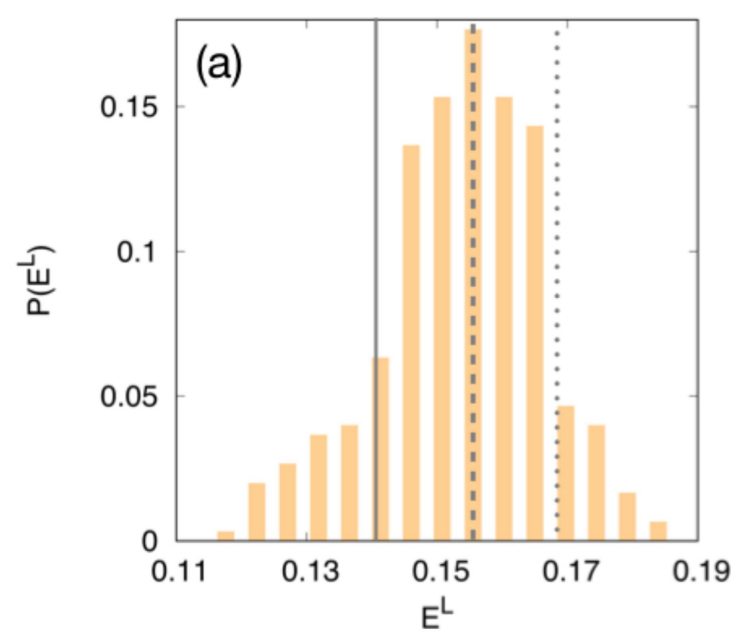

P3 Societal Challenges

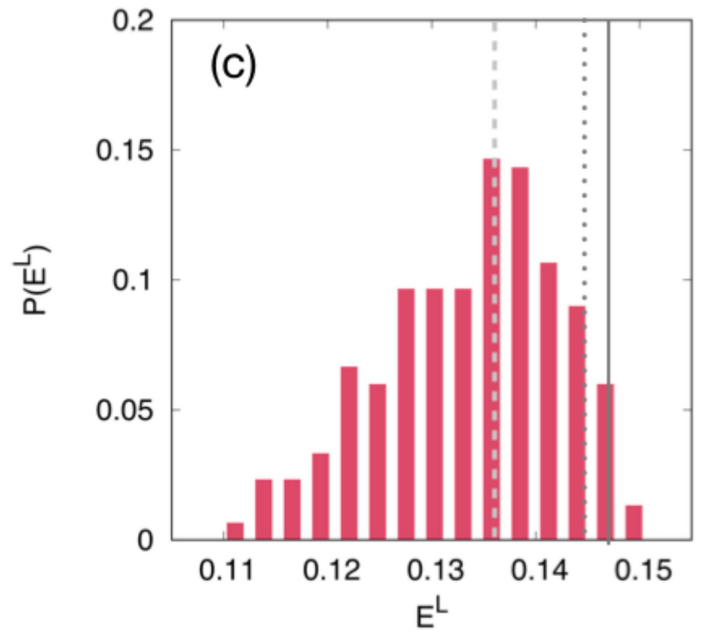

P2 Industrial Leadership

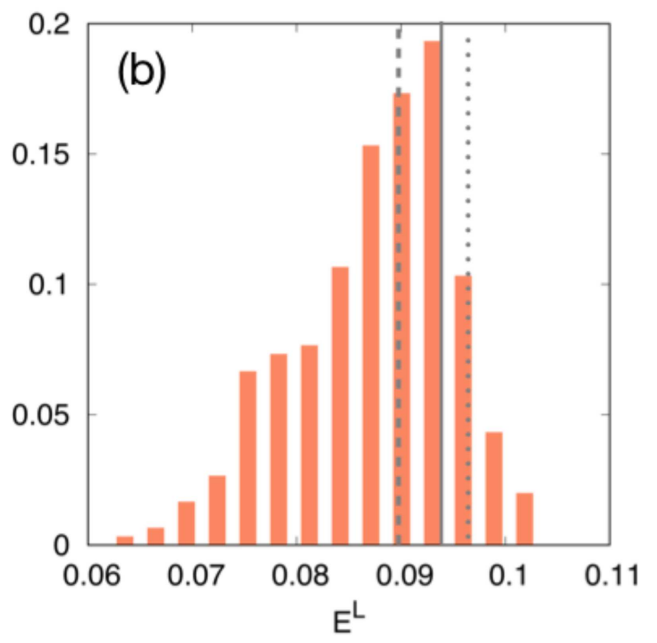

Aggregated network

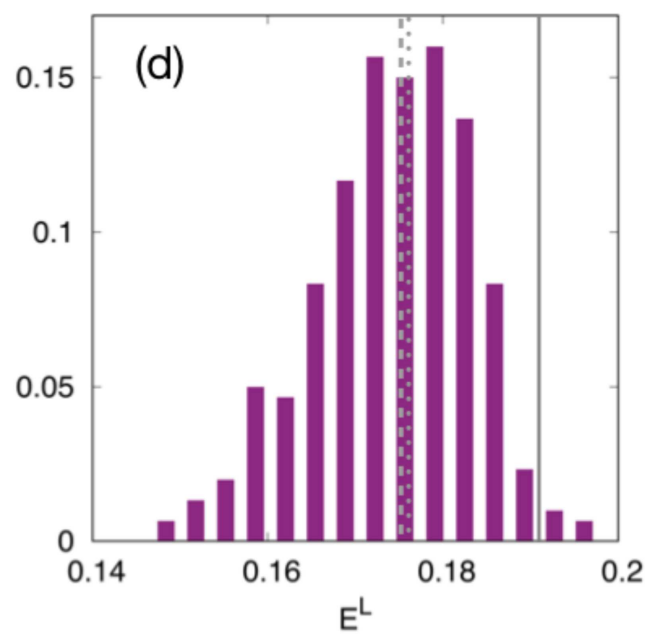

FIG. 3. Values of the local efficiency, $E^{L}$, for the original networks (dotted line), the collaboration graphs without UK institutions (solid line), and the median of the values for $E^{L}$ obtained when randomly removing a similar number of nodes to those removed after BREXIT in the corresponding networks. We also show the histogram of these latter values for a set of 500 networks for which a random deletion of nodes have been implemented. The plots correspond to program P1 (a), P2 (b), P3 (c), and the aggregated network (d).

The comparison has been performed as follows: for each network (BREXIT or each in the random set), we obtain the community partition and compute three descriptors: the number of communities, the average size of the communities, and the standard deviation of the communities size. Then, we have the statistical distribution median (from the random set of networks) and the value of BREXIT partition, for each of the three descriptors. Finally, we compute the statistical significances to compare the BREXIT partition to the 


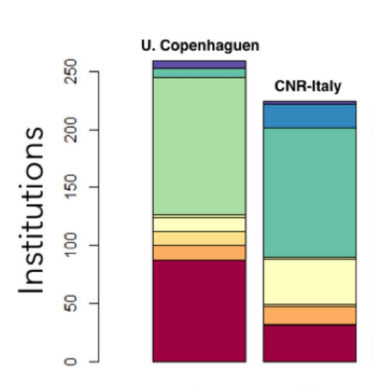

C1

$\mathrm{C} 2$

\section{P1 Excellent Science}
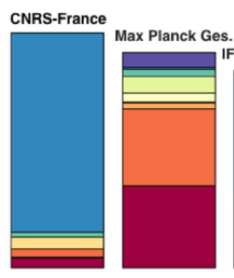

C4

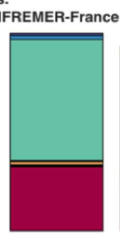

c5

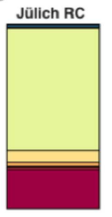

C6

Communities Univ. Leiden

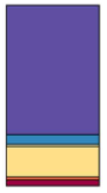

C7

\section{P2 Industrial Leadership}

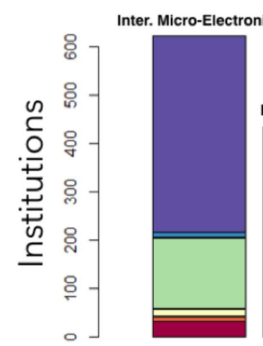

C1

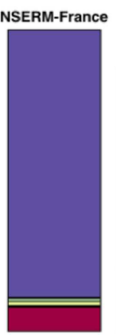

C1

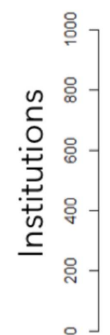

Infineon Tech.

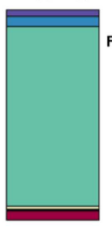

$\mathrm{C} 2$

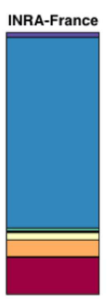

C2

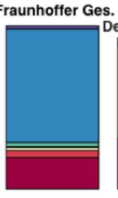

C3

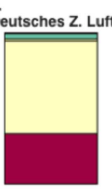

C4

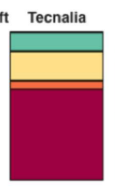

C5

Communities

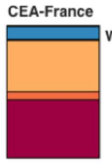

C6

\section{P3 Societal Challenges}

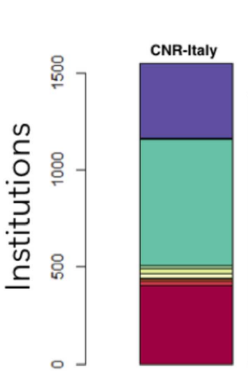

C1

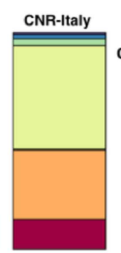

C3

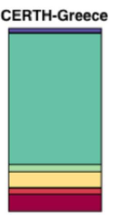

C4

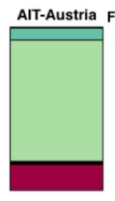

c.5

Communities

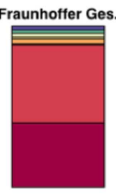

C6
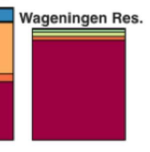

C7

\section{Aggregated network}

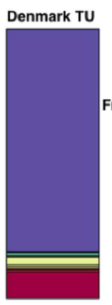

$\mathrm{C} 2$

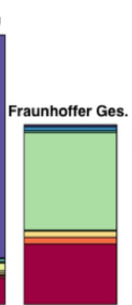

C3

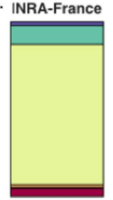

$\mathrm{C} 4$

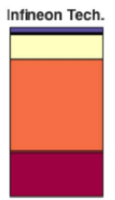

C5

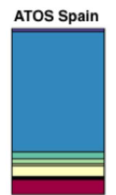

C6

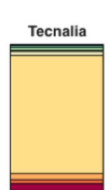

C7

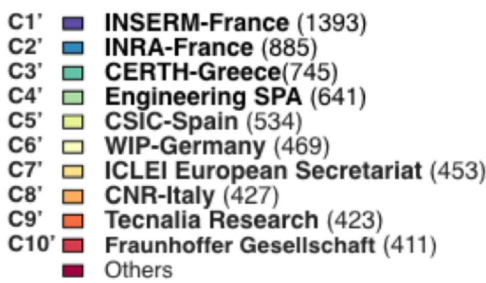

C1' $\square$ EPF Lausanne (399)

C2' $\square$ CNRS-France (349)

C3' $\square$ CNR-Italy (290)

C4' $\square$ U. Copenhaguen (234)

C5' $\square$ Jülich RC (197)

C6' $\square$ UC London (168)

C7' $\square$ U. Birmingham (157)

C8' $\square$ CSIC-Spain (151)

C8' ' CSIC-Spain (151)

C10' $\square$ UC Dublin (128)

Others

C1' Inter. Micro-Electronica (631)

C2' $\square$ Fraunhoffer Gesellschaft (476)

C3' $\square$ Infineon Tech. (451)

C4' $\square$ Atos Spain (321)

C5' $\square$ Acond. Tarrasense (318)

C6' Deutsches Zentrum für Luft (315)

C7' $\square$ TWI Lmtd. (314)

C8' $\square$ CEA-France (304)

C9' $\square$ VTT-Finland (278

C10' Stiftelsen Sintef (269)

- Others

FIG. 4. Size of the seven largest communities $\left(C^{\alpha}\right)$ in the four collaboration networks after BREXIT. The color code in the bar plots shows the composition of the former communities in terms of the ten largest communities in the four original networks $\left(C^{\alpha \prime}\right)$. The names describing the new and original communities refer to those institutions displaying the largest number of collaborations within the corresponding community. 


\section{$W^{\text {BREXIT }} \quad$ W $^{\text {random }}$}

- - - - - - -

P1 Excellent Science

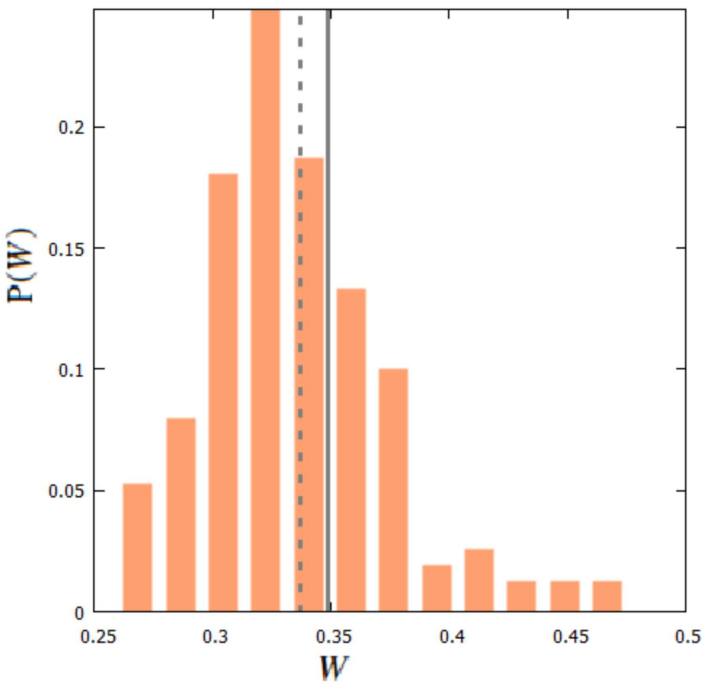

P3 Societal Challenges

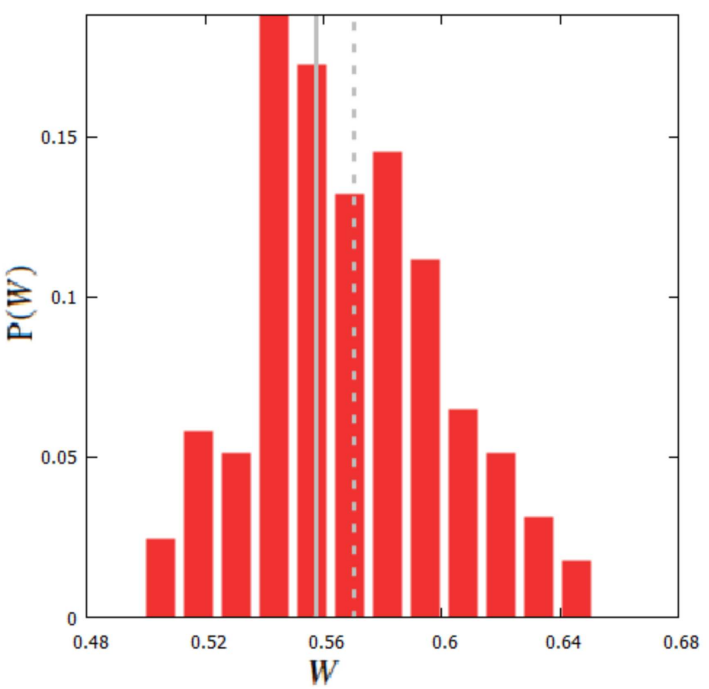

P2 Industrial Leadership

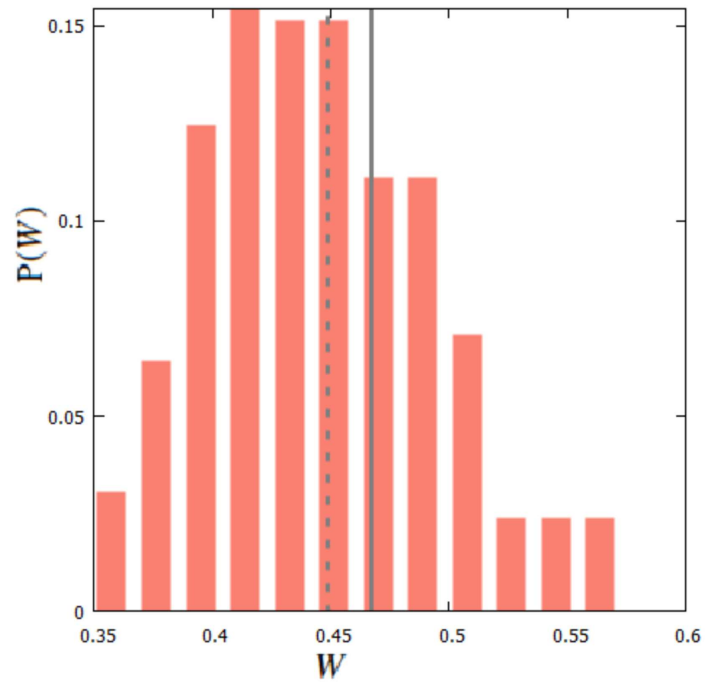

Aggregated network

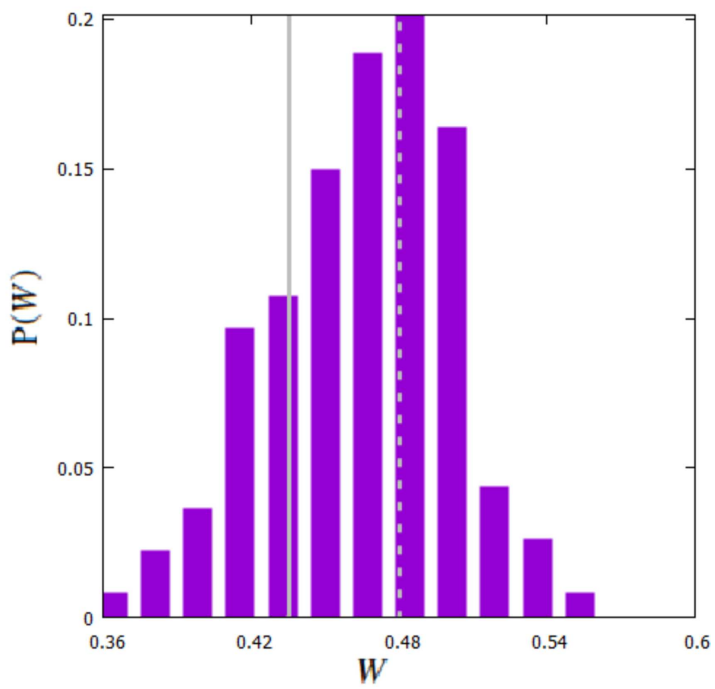

FIG. 5. Values of Wallace index computed comparing the BREXIT network and the former network (WREEIT, represented by solid lines) and histograms for the Wallace index values comparing the set of random networks and the former network ( $W^{\text {Random }}$ is the median of the histograms and is represented by dashed lines), for each of the three research programs and the aggregated network. The random set is composed of 500 graphs.

random set of networks partitions, for each program and the aggregated network. This additional analysis provides a more detailed idea about the nature of community partitions we are computing the Wallace Index for.
Looking at Table $V$, we can conclude that networks without UK nodes are consistent with the statistical sample regarding the communities size. Regarding the average size, we can observe some statistical differences for $\mathrm{P} 1$, in which the community partition 
TABLE V. Comparison table for the number of communities in partition ( $N_{B R E X I T}$, $N_{\text {Random }}$ ), the average size of communities ( $\langle\text { Size }\rangle_{B R E X I T},\langle\text { Size }\rangle_{\text {Random }}$ ), and the dispersion of communities size distribution $\left(\sigma_{B R E X I T}^{\text {size }}, \sigma_{\text {Random }}^{\text {size }}\right)$. We present values for the median of the random set of networks, the network without UK nodes, and the significance of this value, for each of the three programs and the aggregated network.

\begin{tabular}{lcccc}
\hline \hline Network & P1 & P2 & P3 & Aggregated \\
\hline$N_{\text {BREXIT }}$ & 26 & 37 & 27 & 35 \\
$N_{\text {Random }}$ & 24 & 37 & 31 & 35 \\
$s f c\left(N_{\text {BREXIT }}\right)$ & 0.52 & 0.0 & 0.94 & 0.0 \\
$\langle\text { Size }\rangle_{\text {BREXIT }}$ & 109.0 & 153.9 & 321.1 & 398.0 \\
$\langle\text { Size }\rangle_{\text {Random }}$ & 120.0 & 149.9 & 280.0 & 391.7 \\
sfc $\left(\langle\text { Size }\rangle_{\text {BREXIT }}\right)$ & 0.87 & 0.41 & 0.94 & 0.16 \\
$\sigma_{\text {BREX }}^{\text {size }}$ & 64.0 & 122.2 & 247.9 & 398.8 \\
$\sigma_{\text {Random }}^{\text {size }}$ & 65.8 & 110.4 & 238.5 & 403.4 \\
sfc $\left(\sigma_{\text {BREXIT }}^{\text {size }}\right)$ & 0.16 & 0.7 & 0.44 & 0.4 \\
\hline \hline
\end{tabular}

becomes slightly more disaggregated when removing UK nodes than random nodes. On the other hand, for P3, the effect is the opposite, the community partition for the networks without UK nodes is more compact than the partitions of random networks. This implies that the analysis for P3, in terms of the Wallace Index, is not as meaningful as for the other networks, since the Wallace Index has a strong dependence on the number and size of communities in the compared partitions.

\section{DISCUSSION AND CONCLUSIONS}

We have analyzed the efficiency and robustness under BREXIT of the European research collaboration networks formed through H2020 projects. Our analysis has been carried out at global, local, and mesoscopic levels by measuring global and local efficiency and the structure of communities, respectively. The collaboration networks analyzed correspond to the three main H2020 programs (Excellent Science, Industrial Leadership, and Societal Challenges) as well as to the aggregation of them.

The main conclusion is that this European R\&D network is mature enough to resist a rather dramatic event like BREXIT. With the EU support, the different entities participating in H2020 have woven a dense web of interrelations with high connectivity (despite that we have considered that two nodes are related only if one of them is the coordinator of the common project), efficiency, and robustness under a targeted intervention such as BREXIT. We have studied how those different network metrics vary when UK institutions are removed and we have compared them with null models in which the same quantity of nodes is randomly removed from the network. Some differences appear as a function of the metric and of the program studied.

In terms of global efficiency, the Excellent Science program is the one that suffers a bigger negative impact, reflecting the importance of the UK to it as revealed by the highly central role played by UK institutions in this specific program. On the contrary, in the Industrial Leadership program, the deletion of UK nodes has a smaller impact than the elimination of the same number of nodes at random. In the case of local efficiency, we also find a significant decrease for the Excellent Science program while, for the Societal Challenges program and the aggregated networks, this local efficiency is larger in the network without the UK nodes than when removing nodes at random, and also bigger than in the original networks. This result indicates that, in these networks, UK nodes provide an important connectivity at the local level or, in other words, that their neighbors have a scarce connectivity (lower than the average) between them. Finally, at the mesoscale, we have observed that BREXIT does not create a large destruction of the R\&D communities formed under $\mathrm{H} 2020$, despite its effect being different depending on the program, the larger being in the Excellent Science case.

In summary, we can conclude that, from a network perspective, BREXIT will not cause a dramatic effect in the European R\&D ecosystem created under the H2020 program, thanks to the dense and robust interrelations established among many entities of the different European countries.

\section{ACKNOWLEDGMENTS}

We acknowledge financial support from the Spanish Ministerio de Economía y Competitividad through Project Nos. FIS201787519-P and PGC2018-094684-B-C22 and from the Departamento de Industria e Innovación del Gobierno de Aragón y Fondo Social Europeo through Project Nos. E30_17R (COMPHYS group) and E36_17R (FENOL group).

\section{DATA AVAILABILITY}

The data that support the findings of this study are available from the corresponding author upon reasonable request.

\section{REFERENCES}

${ }^{1}$ M. E. J. Newman, Networks: An Introduction (Oxford University Press, Oxford, 2010).

${ }^{2}$ E. Estrada, The Structure of Complex Networks: Theory and Applications (Oxford University Press, Oxford, 2011).

${ }^{3}$ V. Latora, V. Nicosia, and G. Russo, Complex Networks: Principles, Methods and Applications (Cambridge University Press, Cambridge, 2017).

${ }^{4}$ R. Albert and A.-L. Barabási, "Statistical mechanics of complex networks," Rev. Mod. Phys. 74, 47 (2002).

${ }^{5}$ S. N. Dorogovtsev, A. V. Goltsev, and J. F. F. Mendes, "Critical phenomena in complex networks," Rev. Mod. Phys. 80, 1275 (2008).

${ }^{6}$ A. Barrat and A. Vespignani, Dynamical Processes on Complex Networks (Cambridge University Press, Cambridge, 2008).

${ }^{7}$ M. A. Porter and J. M. Gleeson, Dynamical Systems on Networks: A Tutorial (Springer, Berlin, 2016).

${ }^{8}$ D. Stauffer and A. Aharony, Introduction to Percolation Theory (Taylor and Francis, London, 1994).

${ }^{9}$ R. Albert, H. Jeong, and A.-L. Barabási, "Attack and error tolerance of complex networks," Nature 406, 378 (2000).

${ }^{10}$ R. Cohen, K. Erez, and S. Havlin, "Resilience of the Internet to random breakdowns,” Phys. Rev. Lett. 85, 4626 (2000).

${ }^{11}$ D. S. Callaway, M. E. J. Newman, S. H. Strogatz., and D. J. Watts, "Network robustness and fragility: Percolation on random graphs," Phys. Rev. Lett. 85, 5468-5471 (2000).

${ }^{12}$ R. Cohen, K. Erez, D. Ben-Avraham, and S. Havlin, "Breakdown of the Internet under intentional attack,” Phys. Rev. Lett. 86, 3682 (2001).

${ }^{13}$ I. Dobson, B. A. Carreras, V. E. Lynch, and D. E. Newman, "Complex systems analysis of series of blackouts: Cascading failure, critical points, and selforganization," Chaos 17, 026103 (2007). 
${ }^{14}$ D. J. Watts, "A simple model of global cascades on random networks," Proc. Natl. Acad. Sci. U.S.A. 99, 5766 (2002).

${ }^{15}$ K. I. Goh, D.-S. Lee, B. Kahng, and D. Kim, "Sandpile on scale-free networks," Phys. Rev. Lett. 91, 148701 (2003).

${ }^{16}$ A. E. Motter and Y.-Ch. Lai, "Cascade-based attacks on complex networks," Phys. Rev. E. 66, 065102 (2002).

${ }^{17}$ R. Albert, I. Albert, and G. L. Nakarado, "Structural vulnerability of the North American power grid,” Phys. Rev. E 69, R025103 (2004).

${ }^{18} \mathrm{~J}$. Gómez-Gardenes, P. Echenique, and Y. Moreno, "Immunization of real complex communication networks," Eur. Phys. J. B 49, 259-264 (2006).

${ }^{19}$ R. V. Solé, M. Rosas-Casals, B. Corominas-Murtra, and S. Valverde, "Robustness of the European power grids under intentional attack," Phys. Rev. E 77, 026102 (2008).

${ }^{20}$ S. Faci-Lázaro, J. Soriano, and J. Gómez-Gardeñes, "Impact of targeted attack on the spontaneous activity in spatial and biologically-inspired neuronal networks," Chaos 29, 083126 (2019).

${ }^{21}$ T. Tanizawa, G. Paul, R. Cohen, S. Havlin, and H. E. Stanley, "Optimization of network robustness to waves of targeted and random attacks," Phys. Rev. E 71, 047101 (2005).

${ }^{22}$ G. Paul, S. Sreenivas, and H. E. Stanley, "Resilience of complex networks to random breakdown," Phys. Rev. E 72, 056130 (2005).

${ }^{23} \mathrm{P}$. Echenique, J. Gómez-Gardeñes, Y. Moreno, and A. Vázquez, "Distance-d covering problems in scale-free networks with degree correlations," Phys. Rev. E 71, 035102 (2005).

${ }^{24}$ G. Paul, T. Tanizawa, S. Havlin, and H. E. Stanley, "Optimization of robustness of complex networks,” Eur. Phys. J. B 38, 187 (2004).

${ }^{25}$ A. E. Motter, "Cascade control and defense in complex networks," Phys. Rev. Lett. 93, 098701 (2004)

${ }^{26}$ S. Boccaletti, G. Bianconi, R. Criado, C. I. del Genio, J. Gómez-Gardeñes, M. Romance, I. Sendiña-Nadal, Z. Wang, and M. Zanin, "The structure and dynamics of multilayer networks," Phys. Rep. 544, 1-122 (2014).

${ }^{27}$ S. V. Buldyrev, R. Parshani, G. Paul, H. E. Stanley, and S. Havlin, "Catastrophic cascade of failures in interdependent networks," Nature 464, 08932 (2010).

${ }^{28}$ R. Parshani, S. V. Buldyrev, and S. Havlin, "Interdependent networks: Reducing the coupling strength leads to a change from a first to second order percolation transition," Phys. Rev. Lett. 105, 048701 (2010).

${ }^{29}$ R. M. D’Souza, J. Gómez-Gardeñes, J. Nagler, and A. Arenas, "Explosive phenomena in complex networks," Adv. Phys. 68, 123-223 (2019).

${ }^{30} \mathrm{~J}$. Gao, S. V. Buldyrev, S. Havlin, and H. E. Stanley, "Robustness of a network of networks," Phys. Rev. Lett. 107, 195701 (2011).

${ }^{31}$ X. Huang, J. Gao, S. V. Buldyrev, S. Havlin, and H. E. Stanley, "Robustness of interdependent networks under targeted attacks," Phys. Rev. E 83, 065101 (2011).

${ }^{32}$ Ch.D. Brummitt, R. M. D'Souza, and E. A. Leicht, "Suppressing cascades of load in interdependent networks," Proc. Natl. Acad. Sci. U.S.A. 109, E680 (2012).

${ }^{33}$ A. Cardillo, M. Zanin, J. Gómez-Gardeñes, M. Romance, A. J. G. del Amo, and S. Boccaletti, "Modeling the multi-layer nature of the European Air Transport Network: Resilience and passengers re-scheduling under random failures," Eur. Phys. J. Special Topics 215, 23-33 (2013).
${ }^{34}$ C. M. Schneider, N. Yazdani, N. A. M. Araújo, S. Havlin, and H. J. Herrmann, "Towards designing robust coupled networks," Sci. Rep. 3, 1969 (2013).

${ }^{35}$ J. Nunez-Ferrer and D. Rinaldi, "The impact of Brexit on the EU budget: A non catastrophic event," CEPS Policy Brief 347, 1 (2016).

${ }^{36} \mathrm{~A}$. Matthews, "The potential implications of a Brexit for future EU agri-food policies," EuroChoices 15, 17-23 (2016).

${ }^{37} \mathrm{~F}$. Kazzazi et al., "Evaluating the impact of Brexit on the pharmaceutical industry," J. Pharm. Policy Pract. 10, 32 (2017).

${ }^{38}$ S. G. Enger and F. Castellacci, "Who gets Horizon 2020 research grants? Propensity to apply and probability to succeed in a two-step analysis," Scientometrics 109, 1611 (2016).

${ }^{39}$ B. Salmelin, "The Horizon 2020 framework and open innovation ecosystems," J. Innov. Manage. 1, 4 (2013).

${ }^{40} \mathrm{R}$. Veugelers et al., "The impact of Horizon 2020 on innovation in Europe," Intereconomics 50, 4 (2015).

${ }^{41}$ A. Protogerou, Y. Caloghirou, and E. Siokas, "Policy-driven collaborative research networks in Europe,” Econ. Innov. New Technol. 19, 349 (2010).

${ }^{42}$ European Commission, Directorate-General for Research and Innovation, Study on Network Analysis of the 7th Framework Programme Participation, Final Report, 2015. doi:10.2777/50633.

${ }^{43}$ A. G. Avedas et al., Structuring Effects of Community Research-The Impact of the Framework Programme on Research and Technological Development (RTD) on Network Formation, Final Report to the European Commission, 2009.

${ }^{44}$ B. Heller-Schuh et al., Analysis of Networks in European Framework Programmes 1984-2006 (Joint Research Centre, Seville, 2011).

${ }^{45}$ S. Breschi, L. Cassi, F. Malerba, and N. S. Vonortas, "Networked research: European policy intervention in ICTs," Technol. Anal. Strategic Manag. 21, 833 (2009).

${ }^{46} \mathrm{D}$. Defazio, A. Lockett, and M. Wright, "Funding incentives, collaborative dynamics and scientific productivity: Evidence from the EU framework program," Res. Policy 38, 293 (2009).

${ }^{47} \mathrm{See}$ https://data.europa.eu/euodp/en/data/dataset/cordisH2020projects for more information about the list of projects and the institutions involved.

${ }^{48} \mathrm{See} \mathrm{http://research.kampal.com/} \mathrm{for} \mathrm{more} \mathrm{information} \mathrm{about} \mathrm{the} \mathrm{collaboration}$ networks for each program.

${ }^{49} \mathrm{~V}$. Latora and M. Marchiori, "Efficient behavior of small-world networks," Phys. Rev. Lett. 87, 198701 (2001).

${ }^{50} \mathrm{~V}$. Latora and M. Marchiori, "Economic small-world behavior in weighted networks," Eur. Phys. J. B 32, 249-263 (2002).

${ }^{51}$ S. Fortunato, "Community detection in graphs," Phys. Rep. 486, 75 (2010).

${ }^{52} \mathrm{~V}$. D. Blondel, J. L. Guillaume, R. Lambiotte, and E. Lefebvre, "Fast unfolding of communities in large networks," J. Stat. Mech. 2008, P10008 (2008).

${ }^{53}$ M. E. J. Newman, "Modularity and community structure in networks," Proc. Natl. Acad. Sci. U.S.A. 103, 8577 (2006).

${ }^{54} \mathrm{D}$. L. Wallace, "A method for comparing two hierarchical clusterings: Comment," J. Am. Stat. Assoc. 78, 569-576 (1983).

${ }^{55} \mathrm{~S}$. Kumar and J. M. Jan, "Mapping research collaborations in the business and management field in Malaysia, 1980-2010," Scientometrics 97, 1-27 (2013).

${ }^{56} \mathrm{~J}$. Bian et al., "Social network analysis of biomedical research collaboration networks in a CTSA institution," J. Biomed. Inform. 52, 130-140 (2014). 